\title{
Variations in radial maze performance under different levels of food and water deprivation
}

\author{
ROBERT H. I. DALE and WILLIAM A. ROBERTS \\ University of Western Ontario, London, Ontario, Canada
}

\begin{abstract}
Four groups of rats were tested on an eight-arm radial maze under a free-choice procedure. The subjects were maintained at either $80 \%$ or $100 \%$ of their preexperimental free-feeding weights through restricted access to either food or water. Water-deprived subjects received water in the maze; food-deprived subjects received food. Water-deprived subjects learned the task faster than food-deprived subjects. The four groups developed different response patterns. These were measured by the mean transition size, the average angular distance (in $45^{\circ}$ units) between consecutively chosen arms. Rats foraging for food and water developed different search strategies, with water-deprived subjects exhibiting lower mean transition sizes. When the subjects were given three consecutive trials, 2 min apart, choice accuracy declined across trials, although performance on the last two trials improved across days. The groups' mean transition sizes remained different, and were constant over trials and days. Thus, the test procedures differentially affected choice accuracy and response patterning.
\end{abstract}

The eight-arm radial maze consists of eight arms projecting outward radially from a central platform, like the spokes of a wheel (Olton \& Samuelson, 1976). Under a free-choice procedure, food-deprived rats are released on the center platform and are allowed to collect a small piece of food from the end of each arm, in any order. Subjects rapidly attain high choice accuracy, learning to collect all eight pieces of food in only eight or nine choices. Although response strategies, such as choosing adjacent arms in a clockwise order, are not necessary for accurate choice on the radial maze (Olton, Collison, \& Werz, 1977; Roberts \& Dale, 1981), response patterning is prevalent in radial maze research (Dale, 1982; Einon, 1980; Foreman, 1985). In the present experiment, we examined both choice accuracy and response patterns on the radial maze.

Given the emphasis on the ecological relevance of the radial maze (Olton, Handelmann, \& Walker, 1981) and the contribution of natural foraging strategies to radial maze performance (Olton, 1982), it is surprising that the influence of motivational/incentive variables on radial maze performance has received very little attention. For example, only one experiment (Gaffan \& Davies, 1981) has compared the performances of food-deprived and water-deprived rats. Gaffan and Davies found no important performance differences between food-deprived and water-deprived subjects; however, they did not match

The Natural Sciences and Engineering Research Council of Canada supported this research through a grant to $W$. A. Roberts and a postgraduate scholarship to Robert Dale. Preliminary data were presented at the 38th annual meeting of the Canadian Psychological Association, Vancouver, Canada, June 1977. Reprints may be obtained from Robert H. I. Dale, Department of Psychology, Southeastern Louisiana University, P. O. Box 690, University Station, Hammond, LA 70402. their subjects for body-weight loss. This makes their results difficult to interpret, especially in light of previous research in which variations in maze performance initially attributed to group differences in food/water deprivation (Petrinovitch \& Bolles, 1954) were later found to result from differences in body-weight loss (Bolles \& Petrinovitch, 1956). In the present experiment, we examined the radial maze behavior of food-deprived and water-deprived subjects that were matched for body weight loss.

Among the numerous studies demonstrating differences in behavior patterns between subjects responding for food reinforcement and those responding for water reinforcement (see Hogan \& Roper, 1978), several are particularly relevant to radial maze research (Petrinovitch \& Bolles, 1954; Reberg, Mann, \& Innis, 1977; Timberlake, 1983). These studies indicate that water-deprived subjects tend to return to, or remain near, previously visited water sources, whereas food-deprived subjects tend to move away from previously visited food sources. Petrinovitch and Bolles (1954) and Timberlake (1983) took an ecological perspective, relating such behavioral predispositions to the relative stability of water resources and the variability of food resources in the rat's natural environment. Because the radial maze permits wide variations in response patterning without changes in choice accuracy (Foreman, 1985; Olton et al., 1977), groups of subjects tested under different motivational conditions might learn the radial maze task equally well, but exhibit different response patterns.

The experiment presented here examined choice accuracy and response patterning when subjects deprived of either food or water searched for the corresponding reward on the radial maze. A $2 \times 2$ factorial design was used to dissociate the effects of type and degree of depri- 
vation. Different groups of subjects were maintained at $80 \%$ or $100 \%$ of their preexperimental free-feeding weights by restricting their access to either food or water. Choice accuracy was measured in two ways: by the number of different arms selected during a subject's first eight choices on a trial, and by the total number of errors (arm repetitions) made before each subject attained a standard performance criterion. The former measure is commonly used to describe radial maze performance (Olton \& Samuelson, 1976), and allows the groups' choice accuracies to be compared at different points during testing. The latter measure provides a more sensitive summary measure of acquisition rate (Dale, 1982; Petrinovitch \& Bolles, 1954). Response patterns were analyzed by calculating the mean transition size, or the average angular distance between pairs of consecutively chosen arms, measured in $45^{\circ}$ units, so that adjacent arms of the maze were 1 unit apart and opposite arms of the maze were 4 units apart. The mean transition size provides a summary description of how sharply a subject turns between choices on the radial maze (Dale, 1982).

After some pretraining, each subject received one trial on each of 10 consecutive days, followed by three trials (spaced 2 min apart) on each of 4 consecutive days. Under the three-trial procedure, choice accuracy might be expected to decline across trials because of proactive interference in spatial memory, and a compensatory increase in response patterning might be expected to occur (Roberts \& Dale, 1981). This manipulation provided the opportunity to observe, across deprivation conditions, the relationship between choice accuracy and response patterning.

\section{METHOD}

\section{Subjects}

The subjects were 58 experimentally naive male hooded rats, 3 months old, obtained from Canadian Breeding Farms and Laboratories in Montreal, Canada. They were individually housed at $20^{\circ}-22^{\circ} \mathrm{C}$ under a $12 \mathrm{~h} / 12 \mathrm{~h}$ light/dark cycle. The housing room was brightly illuminated by fluorescent ceiling lamps during the light phase of the cycle, and dimly illuminated by a $40-\mathrm{W}$ desk lamp during the dark phase of the cycle.

\section{Apparatus}

An elevated open field and two elevated straight runways were used in pretraining. An eight-arm radial maze was used in the test sessions. All were constructed of 1.2-cm- and 2.4-cm-thick wood. The open field was $91 \mathrm{~cm} \times 67 \mathrm{~cm}$, with a $4.5-\mathrm{cm}$-high wooden border. A small black plastic cup, made from a Kodak $35-\mathrm{mm}$ film container, was located at the center of the open field. The two straight runways were the same size, $122 \mathrm{~cm} \times 9 \mathrm{~cm}$, each with a small plastic cup $4 \mathrm{~cm}$ from each of its ends. The radial maze consisted of eight identical arms, spaced $45^{\circ}$ apart, projecting radially outward from a central octagonal choice area. The octagonal center platform was $31 \mathrm{~cm}$ wide; each arm was $76 \mathrm{~cm}$ long and $8 \mathrm{~cm}$ wide. A small black plastic cup was located $2.5 \mathrm{~cm}$ from the outer end of each arm.

Pretraining and testing were conducted in different rooms. Each subject was tested in one of two rooms that were similar in size $(2.1 \mathrm{~m} \times 3.5 \mathrm{~m})$ and construction. Each room had a brick floor, fluorescent ceiling light fixtures, a door, a sink, a counter, a boarded picture window, and a fan vent in one corner. The pretraining rooms were different in size and shape from the test rooms, and contained a variety of objects. The lighting levels in the pretraining and test rooms were similar to the lighting level in the housing room during the dark phase of the light/dark cycle.

\section{Procedure}

Deprivation conditions. Initially, 48 subjects were divided into four groups $(\mathrm{N}=12)$, matched for their preexperimental freefeeding weights. However, 1 subject in Group Water-100\% and 3 subjects in Group Food-100\% were discarded from the experiment because they failed to make at least eight choices within $15 \mathrm{~min}$ on each of two consecutive trials. They were replaced by 7 additional Food- $100 \%$ subjects and 3 additional Water- $100 \%$ subjects. Four of the Food-100\% replacements and 2 of the Water-100\% replacements completed the experiment. Thus, the experiment was completed by 12 subjects in each of Groups Water-80\% and Food$80 \%$, and by 13 subjects in each of Groups Water-100\% and Food$100 \%$. The subjects in the Group Food-80\% were maintained at constant weights through restricted access to food: about $1 \mathrm{~h}$ after testing, about $15 \mathrm{~g}$ of Purina Rat Chow was placed in each subject's cage. The subjects in Group Food-100\% received about $20 \mathrm{~g}$ of Purina Rat Chow about $1 \mathrm{~h}$ after each daily test session. Both groups had free access to water. The mean weight of the subjects in Group Food-100\% gradually increased from $97 \%$ to $103 \%$ of baseline between the first and last radial maze test days. The subjects in Group Water- $80 \%$ were maintained at constant weights by being restricted to $5 \mathrm{~min}$ daily access to water, about $1 \mathrm{~h}$ after each test session. The subjects in Group Water-100\% were maintained at approximately $100 \%$ of their baseline weights by limiting their home-cage access to water to $15 \mathrm{~min}$ daily, about $1 \mathrm{~h}$ after each test session. Both groups had free access to food inside their home cages. The mean weight of the subjects in Group Water-100\% increased from $98 \%$ to $103 \%$ of baseline between the first and last radial maze test days. To establish whether subjects maintained at the same weight through food deprivation and water deprivation were under identical deprivation conditions, home-cage food and water intakes were recorded for 6 subjects in each group. Data were obtained over 9 days for 1 subject in Group Food-100\% and over 11 days for the other 23 subjects.

Pretraining. All pretraining and test sessions were conducted during the dark phase of the light/dark cycle. Each subject was given five 5-min exposures to the open field, usually on consecutive days. The plastic cup in the center of the open field contained $2.5 \mathrm{ml}$ of water for the water-deprived subjects and $2045-\mathrm{mg}$ Noyes food pellets for the food-deprived subjects. Each subject was then given, on consecutive days, two 5-min sessions on a straight runway. The two cups on the runway each contained either $1.2 \mathrm{ml}$ water, for the water-deprived subjects, or $1045-\mathrm{mg}$ Noyes pellets, for the fooddeprived subjects. Thus, the amount of food or water available in each pretraining session approximately matched that earned later on the radial maze test trials.

Testing. Each subject was given one trial on the radial maze on each of 10 consecutive days, followed by three trials on each of the next 4 days. Before a trial, each of the eight cups on the maze was baited with two 45-mg Noyes pellets, for food-deprived subjects, or $0.3 \mathrm{ml}$ water, for water-deprived subjects. On each trial, a subject was placed on the center of the maze and allowed $15 \mathrm{~min}$ to enter all of the arms and then repeat one of them (for reasons unrelated to this report, we were interested in which arm would be repeated). The subjects' initial orientations were varied randomly across trials. During the three-trial sessions, the trials were approximately 2 min apart. The subject was returned to its home cage between trials.

The sequence in which the arms were chosen was recorded manually, after the arms had been numbered from 1 to 8 . A choice was recorded whenever a subject's whole body, up to the base of the tail, was inside the arm; subjects rarely entered this far without continuing to the end of the arm. For 6 subjects in each group, the time required to ingest the reward on an arm (reward collection time) was measured for 3 or 4 choices on each trial. This measure 
was obtained because differences in consumption time may have implications for the search strategies used by food-deprived and water-deprived subjects.

\section{RESULTS}

\section{Trials 1-10}

Choice accuracy. The rate at which each subject mastered the radial maze task was measured by the total number of arms it repeated (not counting the compulsory trial-ending error) before attaining a criterion of two consecutive trials with all eight arms chosen in the minimum of eight choices. One subject in each of Groups Water$80 \%$, Water-100\%, and Food-80\%, and 4 subjects in Group Food-100\%, failed to attain criterion within 10 trials. The total number of errors made by these subjects during the 10 trials was calculated. The mean errors (arm repetitions) to criterion were $11.8,13.2,18.2$, and 22.6 for Groups Water-80\%, Water-100\%, Food-80\%, and Food- $100 \%$, respectively. A two-way analysis of variance (weight $\times$ reward) indicated that only the reinforcer main effect was significant $[F(1,46)=15.86, p<.001]$. There was no effect of weight $[F(1,46)=2.00, p>.1]$ and no weight $\times$ reward interaction $[F(1,46)=0.57$, $p>.1]$. Post hoc tests (Tukey's test, $\alpha=.05$ ) indicated that, for each body weight, the water-deprived group attained criterion faster than the food-deprived group.

Choice accuracy was also measured by an accuracy score, the number of different arms chosen during a subject's first eight choices on a trial. The group mean accuracy scores during the first 10 trials are shown at the top of Figure 1. Note that all four groups of subjects were choosing very accurately by Trials $9-10$ (Figure 1, top, Block 5). A three-way analysis of variance (weight $\times$ reward $\times$ trials) produced significant effects for weight $[F(1,46)=4.06, p<.05]$, reward $[F(1,46)=10.20$, $p<.01]$, and trials $[F(1,46)=38.36, p<.001]$. The weight $\times$ trials interaction was the only significant interaction $[F(9,414)=2.12, p<.05]$; the other interactions did not reach significance at the $p=.10$ level. The weight $x$ trials interaction occurred because the subjects at $80 \%$ body weight did not obtain higher accuracy scores than the subjects at $100 \%$ body weight until the later trials.

Choice sequences. The four groups of subjects displayed different choice sequences. In particular, the waterdeprived subjects made a higher proportion of adjacentarm choices than the food-deprived subjects. This effect was measured by the mean transition size (the average angular distance between consecutively chosen arms, in $45^{\circ}$ units). All transitions between arms were assigned a transition size between 0 and 4 . To equate the samples taken from different subjects and at different points in testing only the first eight choices on each trial (the first seven transitions) were considered. The mean transition sizes for each group, averaged over two-trial blocks, are shown at the bottom of Figure 1. An analysis of variance (weight $\times$ reward $\times$ trials) indicated that the main effects of weight $[F(1,46)=16.88, p<.01]$, reward $[F(1,46)=$
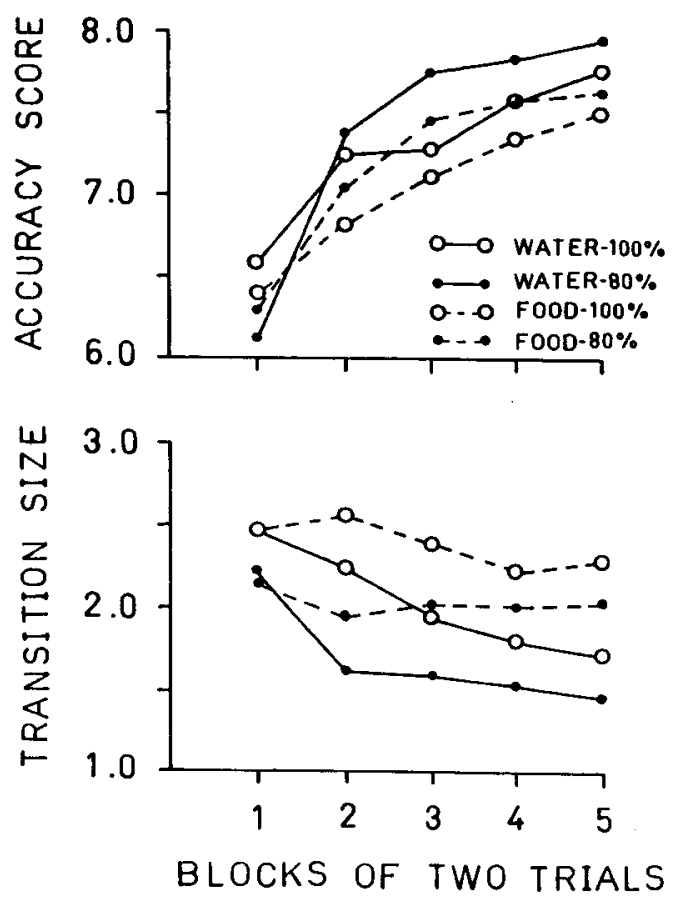

Figure 1. Mean accuracy scores (top) and mean transition sizes (bottom) during Trials 1-10, averaged over two-trial blocks. Groups are identified by their body weights $(80 \%, 100 \%)$ and the substance of which they were deprived (food, water).

$16.71, p<.01]$, and trials $[F(9,414)=11.70, p<.01]$ were significant, as were the weight $\times$ trials $[F(9,414)$ $=2.87, p<.01]$ and reward $\times$ trials $[F(9,414)=5.18$, $p<.01]$ interactions. The other interactions did not reach significance at the $p=.1$ level. The reward $\times$ trials interaction reflects the fact that reward type did not influence mean transition size on the first few trials, but only on later trials. A post hoc test (Tukey's test, $\alpha=.05$ ) indicated that the reward effect was significant on Trial 4 and on Trials 6-10. The weight $\times$ trials interaction reflected a significant weight effect on Trials 2-4 only (Tukey's test, $\alpha=.05$ ).

\section{Trials 11-22}

Choice accuracy. Choice accuracy was measured in terms of the group mean accuracy scores (Figure 2, top). An analysis of variance (weight $x$ reward $x$ days $\times$ trials) conducted on the mean accuracy scores produced significant effects for weight $[F(1,46)=9.44, p<.01]$, day $[F(3,138)=12.44, p<.01]$, and trial $[F(2,92)=$ $56.14, p<.01]$. The reward main effect was not significant $[F(1,46)=1.32, p>.1]$. Of the 11 interactions, only the days $\times$ trials interaction $[F(6,276)=5.99$, $p<.01$ ] was significant at the $p=.1$ level. Thus, the subjects at $80 \%$ of baseline weight performed more accurately than the subjects at $100 \%$ of baseline weight. There was a decrement in choice accuracy across trials within a day, and an improvement in accuracy scores across days. The days $\times$ trials interaction indicates that 

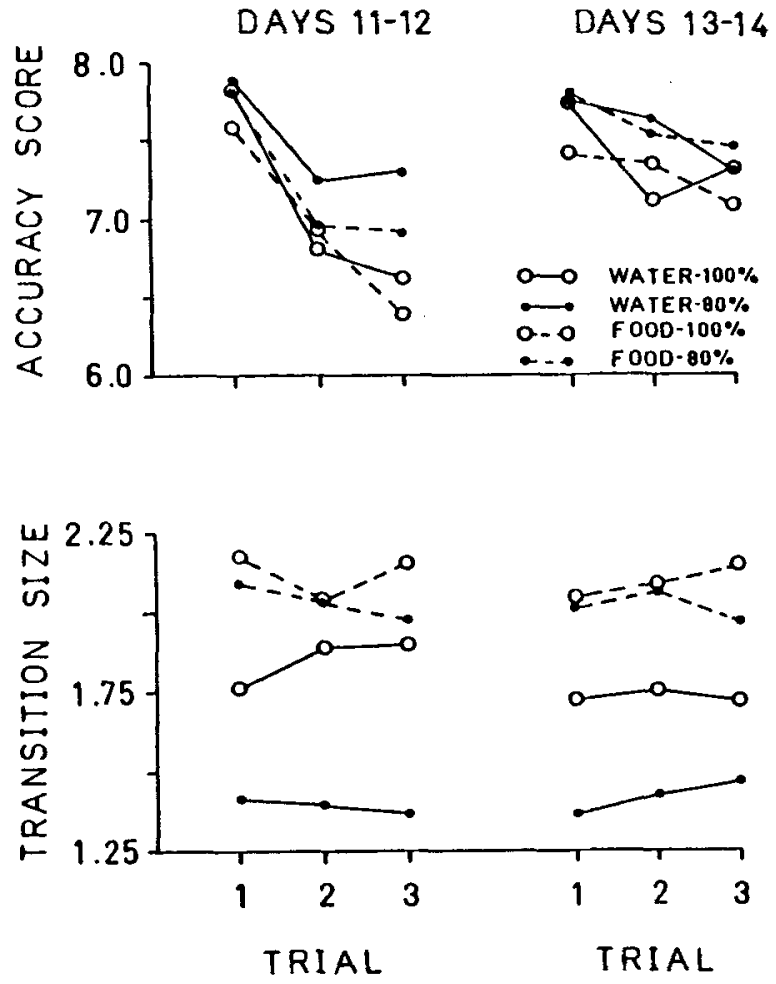

Figure 2. Mean accuracy scores (top) and transition sizes (bottom) on Days 11-12 (left) and Days 13-14 (right), as a function of trials within a day.

the improvement across days occurred only on the second and third trials within a day.

Choice sequences. The bottom half of Figure 2 shows the mean transition sizes for the four groups, as a function of days and trials. In contrast to the accuracy scores, the mean transition sizes were not influenced by days or trials within a day. An analysis of variance (weight $x$ reward $x$ days $x$ trials) produced only two significant effects: weight $[F(1,46)=5.04, p<.05]$ and reward $[F(1,46)=19.63, p<.01]$. None of the other main effects or interactions was significant at the $p=.10$ level. Thus, choice accuracy and choice sequences were differentially affected by the three-trials-a-day procedure.

\section{Deprivation Conditions}

The mean daily food and water intakes for 6 subjects in each group are shown in Table 1. These figures include the food and water consumed during the test sessions. The mean daily home-cage food intake was corrected for spillage. Water spillage appeared to be negligible, but it was not systematically monitored for the subjects with free access to water. Table 1 also shows the mean body weights of the subjects during the monitoring period, both in absolute terms and as a proportion of their preexperimental free-feeding weights. Despite the fact that they were matched for body weights, the subjects in Group Water$80 \%$ ate more $[t(10)=2.60, p<.05]$ and drank less $[t(10)=8.4, p<.01]$ than the subjects in Group Food$80 \%$. The subjects in Group Water-100\% ate less [ $t(10)$ $=3.0, p<.05]$ and drank less $[t(10)=13.7, p<.01]$ than the subjects in Group Food-100\%. Thus, despite their being matched for body weight, the food-deprived and water-deprived subjects were not under identical deprivation conditions.

\section{Reward Collection Times}

Table 1 shows the mean reward collection times for 6 subjects in each group, based on 3 or 4 samples on each trial. The food was collected in about $1 \mathrm{sec}$, the water in 7-10 sec.

\section{DISCUSSION}

There were two major findings. First, both weight loss and deprivation type influenced performance on the radial maze; second, choice accuracy and response sequences were differentially affected by the motivational variables. Choice accuracy during the first 10 trials was primarily determined by reward type, according to both the acquisition criterion and accuracy-score data. Contrary to a simplistic application of Petrinovitch and Bolles's (1954) hypothesis that water-deprived subjects are predisposed to return to water sources whereas food-deprived subjects tend to visit a variety of locations, the water-deprived subjects learned to solve the task more quickly. The accuracy score data also suggested a weak effect of body weight, that is, degree of deprivation (Bolles \& Petrinovitch, 1956).

The transition size measure indicated strong effects of both reward type and body weight, indicating that differences in response patterning were related to both type and degree of deprivation (Bolles \& Petrinovitch, 1956; Petrinovitch \& Bolles, 1954). The reward $\times$ trials interaction observed over Trials $1-10$ for the mean transition

Table 1

Mean Body Weights, Mean Daily Food and Water Intakes*, and Mean Reward Collection Timet for 6 Subjects from Each Group

\begin{tabular}{lcccc}
\hline & \multicolumn{4}{c}{ Group } \\
\cline { 2 - 5 } \multicolumn{1}{c}{ Measure } & Water-80\% & Food-80\% & Water-100\% & Food-100\% \\
\hline Mean body weight $(\mathrm{g})$ & 269 & 269 & 339 & 336 \\
Weight & 79.6 & 80.1 & 99.6 & 99.3 \\
Food intake (g) & 12.4 & 11.4 & 17.1 & 19.5 \\
Water intake (g) & 9.4 & 24.2 & 15.1 & 30.2 \\
Time & 10.1 & 1.1 & 7.5 & 1.0 \\
\hline Note-Weight $=$ percent of baseline weight. Time = reward collection time (in seconds). & *Moni- \\
tored over 9 or 11 days; home cage plus test session intake. †Based on 3 or 4 samples/trial.
\end{tabular}


size measure is important because it indicates that fooddeprived and water-deprived subjects differed in mean turn size not at the start of testing (Figure 1, bottom), but only after some experience on the maze. This makes it impossible to attribute the different response patterns entirely to behavioral predispositions (Petrinovitch \& Bolles, 1954). As in other studies (Reberg et al., 1977; Timberlake, 1983), the behavior observed resulted from an interaction between the subject's motivational state and characteristics of the test situation.

The dissociation of the effects of motivational variables on response patterns and response accuracy was striking during Trials 11-22, when subjects were given three closely spaced trials in each session. The accuracy score was strongly influenced by weight, trial, and day of testing, with a days $\times$ trials interaction. In contrast, the transition size measure reflected only weight and reward effects. In this phase of the experiment, food-deprived and water-deprived subjects chose arms with equal accuracy, but their choice sequences were different and were stable across trials. All four groups, regardless of their response patterning, had high accuracy scores; choice patterns apparently exert a weak influence on choice accuracy, an influence secondary to that of memory processes (Foreman, 1985; Olton et al., 1977; Roberts, 1984).

Several issues with implications for future research have been identified here. First, although body weight and deprivation type were not confounded in the present experiment, type of deprivation and size of reward probably were. That is, it seems likely that $0.3 \mathrm{ml}$ of water and $45 \mathrm{mg}$ of food are, in some sense, different size incentives. One way to unconfound deprivation type and reward size would be to use several different reward sizes for both food-deprived and water-deprived subjects. Surprisingly, there is no evidence that radial maze performance is related to food reward size (Gaffan, Hansel, \& Smith, 1983; Olton \& Samuelson, 1976). Until the effects of reward size on radial maze performance are determined, we cannot be certain whether the effects observed in our experiment were due to the type of deprivation, independent of the size of the reward. Second, deprivation conditions must be carefully defined. The food-deprived and water-deprived subjects were matched for body weight, but consumed different amounts of food and water. Third, the food-deprived and water-deprived subjects spent different amounts of time in the arms of the radial maze. These differences in arm-exposure, or information-processing, time may have induced different memory processes and/or search strategies.

Finally, this experiment must be considered a preliminary demonstration of the systematic influences of motivational/incentive variables on radial maze behavior. It suggests that motivational variables may determine behavior patterns on a task (how the task is solved) as well as the overall accuracy with which the task is completed. Explanations for these effects must await further research.
Adequate explanations for radial maze behavior are likely to be based on a combination of ecological (Olton, 1982; Yoerg \& Kamil, 1982), cognitive (Olton, 1982; Roberts, 1984), and learning-theory (Evenden \& Robbins, 1984; Gaffan et al., 1983) factors.

\section{REFERENCES}

Bolles, R. C., \& Petrinovitch, L. (1956). Body-weight changes and behavioral attributes. Journal of Comparative \& Physiological Psychology, 49, 177-180.

DALE, R. H. I. (1982). Parallel-arm maze performance of sighted and blind rats: Spatial memory and maze structure. Behaviour Analysis Letters, 2, 127-139.

EINON, D. (1980). Spatial memory and response strategies in rats: Age, sex and rearing differences in performance. Quarterly Journal of Experimental Psychology, 32, 473-489.

EVENDEN, J. L., \& RoBBins, T. W. (1984). Win-stay behavior in the rat. Quarterly Journal of Experimental Psychology, 36B, 1-26.

Foreman, N. (1985). Algorithmic responding on the radial maze in rats does not always imply the absence of spatial encoding. Quarterly Journal of Experimental Psychology, 37B, 333-358.

GafFan, E. A., \& Davies, J. (1981). The role of exploration in winshift and win-stay performance on a radial maze. Learning \& Motivation, 12, 282-299.

Gaffan, E. A, Hansel, M. C., \& Smith, L. E. (1983). Does reward depletion influence spatial memory performance? Learning \& Motivation, 14, 58-74.

Hogan, J. A., ROPER, T. J. (1978). A comparison of the properties of different reinforcers. In J. S. Rosenblatt, R. A. Hinde, C. Beer, \& M.-C. Busnel (Eds.), Advances in the study of behavior (Vol. 6, pp. 155-255). New York: Academic Press.

OLToN, D. S. (1982). Spatially organized behaviors in animals: Behavioral and neurological studies. In M. Potegal (Ed.), Spatial abilities: Development and physiological foundations (pp. 335-360). New York: Academic Press.

Olton, D. S., Collison, C., \& Werz, M. A. (1977). Spatial memory and radial arm maze performance in rats. Learning \& Motivation, 8, 289-314.

Olton, D. S., Handelmann, G. E., \& Walker, J. A. (1981). Spatial memory and food searching strategies. In A. C. Kamil \& T. D. Sargent (Eds.), Foraging behavior: Ecological, ethological, and psychological approaches (pp. 333-354). New York: Garland STPM Press.

Olton, D. S., \& SAmuelson, R. J. (1976). Remembrance of places passed: Spatial memory in rats. Journal of Experimental Psychology: Animal Behavior Processes, 2, 97-116.

Petrinovitch, L., \& Bolles, R. C. (1954). Deprivation states and behavioral attributes. Journal of Comparative \& Physiological Psychology, 47, 450-453

REBERG, D., MANN, B., \& INNIS, N. K. (1977). Superstitious behavior for food and water in the rat. Physiology \& Behavior, 19, 803-806.

RoBerTs, W. A. (1984). Some issues in animal spatial memory. In H. L. Roitblat, T. G. Bever, \& H. S. Terrace (Eds.), Animal cognition (pp. 425-443). Hillsdale, NJ: Erlbaum.

Roberts, W. A., \& Dale, R. H. I. (1981). Remembrance of places lasts: Proactive inhibition and patterns of choice in rat spatial memory. Leaming \& Motivation, 12, 261-281.

Timberlake, W. (1983). Rats' responses to a moving object related to food or water: A behavior-systems analysis. Animal Learning \& Behavior, 11, 309-320.

YoERG, S. I., \& KAMIL, A. C. (1982). Response strategies in the radial arm maze: Running around in circles. Animal Learning \& Behavior, $10,530-534$.

(Manuscript received August 14, 1985; revision accepted for publication January $27,1986$. ) 